\title{
$\mathfrak{S} \mathfrak{t} \mathfrak{a} \mathfrak{t} \mathfrak{d t}$
}

Dis

\section{Deutiden Guxitentages.}

$\$ 1$.

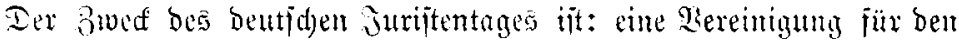

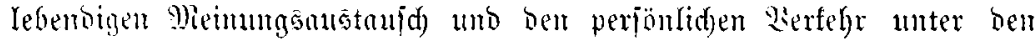

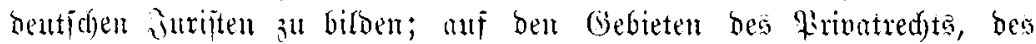

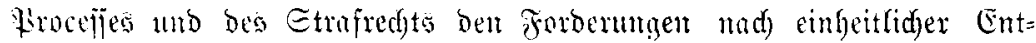
widfelung inmer gröfiere Anerfenmung a verichnffen, bie sindernije,

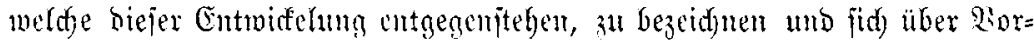

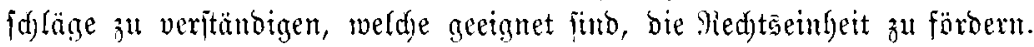

\section{$\S 2$.}

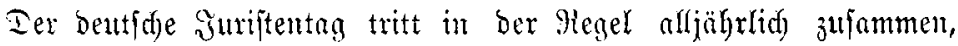

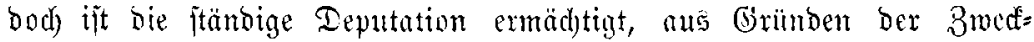

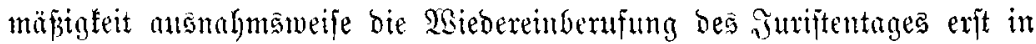

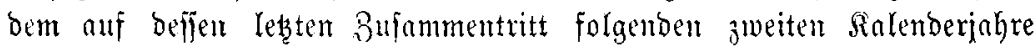
vorzunthment.

\section{$\S 3$.}

Bur Mitgliebldaft beredjtigt find bie beutiden Fidjter, Staatäan=

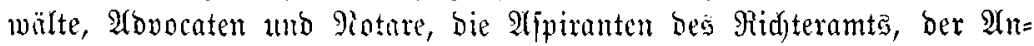
waltfdaft unb bes Rotariats, fowie jeber, ber nad) feinen Randeşgefeben

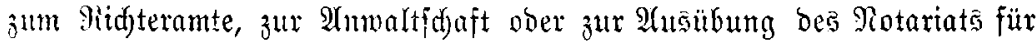
bejübigt erfannt ift, fermer bie Sebrer an ben beutfden frodjidulen, bie Mitglieder ber gelehrten Acabenien, bie Toctoren ber Redte und bie redgtşgelehrten Mitgliebex Der Berwaltungâbehörben. 
$\S 4$.

Die Mityliesfdaft beginnt mit bem Empfange ber Mitgliedsłarte. E:e

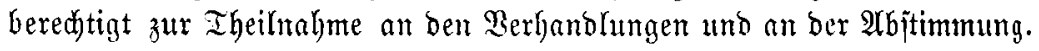

$\S 5$.

Der Beitrag Der Gefelffdjaftsmitglieber beträgt Eerfs Ma Marf jährtit)

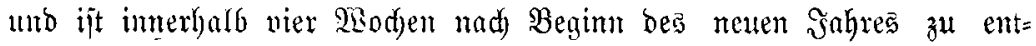
ridften, mibrigenfalfs berfelbe burd) Pojtworjdyuf eingezogen mirb. Rimmt

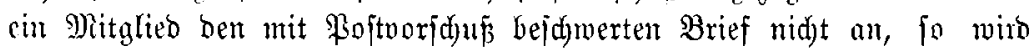

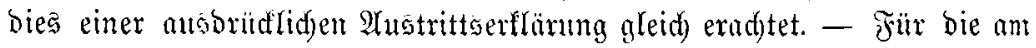

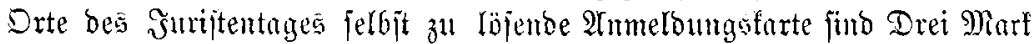
zut entridytert.

$$
\$ 6 .
$$

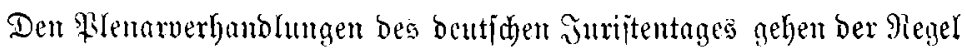

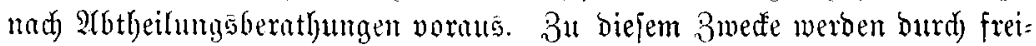
willige Einzzeid)uungen Der Nhitglieber forgenbe vier 2(btheilungen gebithet:

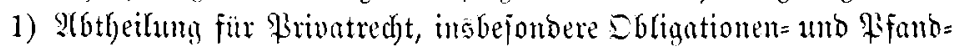

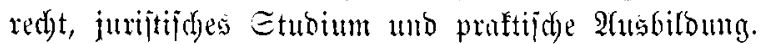

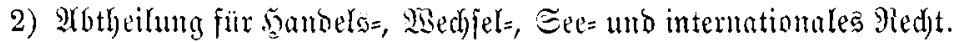

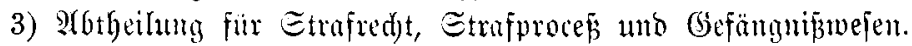

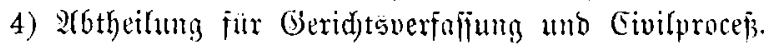

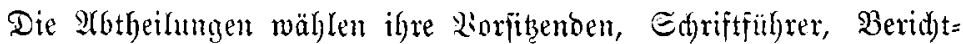

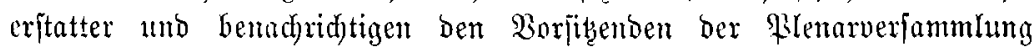
(§ 7), fobuld ihre Berathungen über einzelne Begenfändoe gefdhlofifen

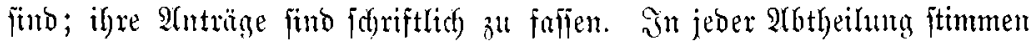
nur Diejenigen mit, meldge jid) in bie betreffente atbtheilung bereits cin= gezeidynet laben.

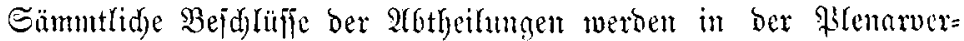

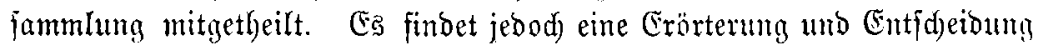
in Blemum nux bann ftatt, menn biejelbe von ber betreffenden arbthei=

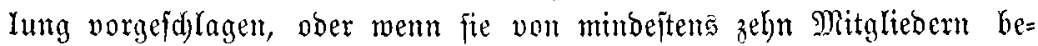

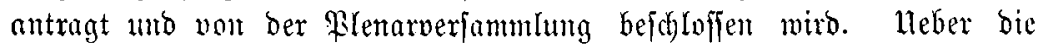
Sorfrage, ob bem von minbeftento zebn s)itgliebern geftellten Âtrage

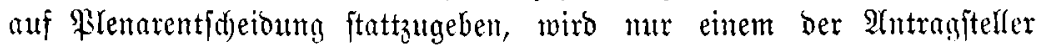
und bem Beridsterftatter bas నిort ertheilt.

\section{$\S 7$.}

Die Berbandlungen ber \$lentroerjammlung leitet ein Borithenber, welder für bie Dauer eines jeben Juriftentageß in bex erften \$lenarver= fammlung burrá) Stimmzettel ober Ilcclamation gewählt wiro. Derfelbe 
benemt zwei bia vier Stelfvertreter und vier Sdyriftü̈lyer. Er beftimmt bie Iagesnrbmung und fann eingelue Begenfände, ohne Borberathung in ben $\mathfrak{A}$ btheilungen $(\$ 6)$, ummittelbar zur \$lenarberathung ftellen. ijt er bejugt, Nichtmitglieber als ßuhörer zuzulafjen.

$\S 8$.

Bei allen Bejhlütjen der ßlenarverfammlung und ber Albtheilungen enticheibet einfache Majorität ber anmejenden Mitglieber, bei allen Mahlen relative Majorität unto im Falle ber Etimmengleitheit bas Roos.

$\S 9$.

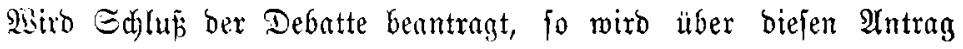
jofort abgeftimmt. In ber Plenarverjammlung find alle Anträge mit

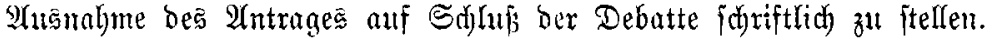

$\$ 10$.

2or bem Edyluffe eines jeben Juriptentages wird von ber glenar=

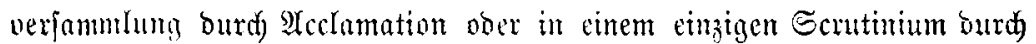
Stimnsettel eine aus neumehn Mitgliebern und den Präjidenten des

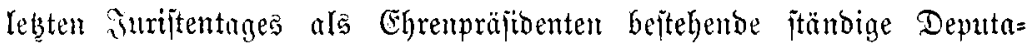
tion gewathlt. Die Sifte ber zur Afclomation vorzufdhlagenben Ferjonen miro burtf) ben Bräjtoenten Der \$lenarperfammlung, feine Stellvertreter umb je zehn von jeder abtheilung gewählte Bertrauengamänner gemein= f(d)aftlidy feftgejtellt.

Tie ftünbige Deputation hat folgende Befugniffe und Dbliegenlgeiten:

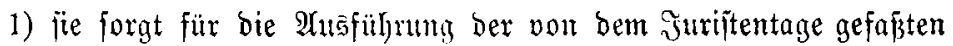
Befd) lüffe, vexanlafit nath eigenem Ermefien ben Dutá ber \$ro= tocolle und Borlagen, berwift bie Bertheilung ber Drudfadjen

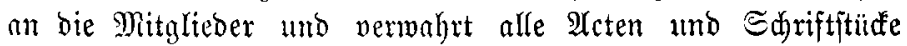
beš Suriftentages;

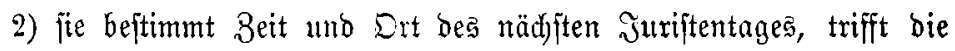
für benfelben nötbigen Sorbereitungen, erläpt bie Einlabungen, ftellt bie vorläuftge Tageșoromung auf, wobei fie in ber Regel nur bie bis zum 31. Mai bes Iaufenden Jahres eingegangenen Borlagen zu berüdfid)tigen hat, unb bereitet $\mathfrak{A b a ̈ n o e r u n g s v o r j a ̆ l a ̈ g e ~}$ in Betreff ber (Befdäfțorbmung für bie \$lenarverfammlung vor;

3) fie nimmt bie Beitritţerflärungen neuer Mitglieber entgegen, fer= tigt bie Mitgliebšlarten aus, empfängt die Beiträge, bejtreitet die R̂agaben und legt ber folgenden Deputation Stedinung; 


\section{VIII}

4) jite ergängt jîd felbjt, falls eins ober mebrere Mitglieber währent bes Gejühätsjahres auşaheiden.

Die Deputation wählt aus igrer Mitte eituen Borj̈tzenden, einett Sdriftfübrer, melder ein von ber Teputation fejtzufetzendes \$aujdoquan= tumt für baare Auslagen erfält, und einen Caffiter. Der Zebtere ijt ver= pflichtet, ber ftändigen Deputation bei ihrem jebesmaligen ôujammentritt

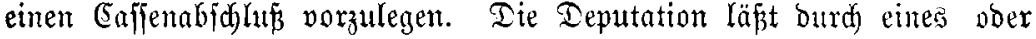
mehrere ihrer Mitglieber bie Red)nung prüfen und bie Caffe revibiren.

Die Deputation bejtimmt Drt uno Beit ihrer 3 ufammenfunft. $8 u \mathfrak{r}$ Gültigfeit ifrer Befdrlüffe ift bie Eintlabung fämmtlidjer Mitglieber, fowie bie Mitwirfung von wenigitens fünf Mitgliebern erforoerlid.

\section{$\S 11$.}

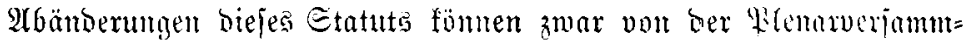

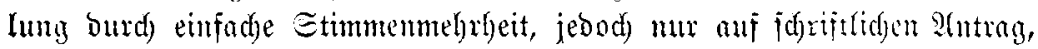
ber vier 9lod)en vor bem 3ujammentritt bes̄ Jurijtentages ber jündigen Deputation (\$ 10) itberreidft worben, befdfoffen weroent. 